\title{
Manifestation of additional frequencies in vortex induced vibrations in the presence of noise
}

\author{
M.S. Aswathy ${ }^{1, \star}$ and Sunetra Sarkar ${ }^{1, \star \star}$ \\ ${ }^{1}$ Department of Aerospace Engineering, Indian Institute of Technology Madras
}

\begin{abstract}
In this study, we examine the effect of random input fluctuations in the mean flow to a circular cylinder undergoing transverse oscillations. A Duffing-Van der pol combined system has been used to model the structure and wake oscillators in the VIV system. We observe that the addition of noise brings in major qualitative and quantitative changes on the structural response of the system compared to the deterministic cases. It has been observed that the stochastic system is always influenced by the presence of structural frequency. In contrast, the system under mean flow condition aligns with the structural frequency, only in the lock-in range. This feature is seen as noise exciting multiple frequencies in the response of the cylinder in the pre lock-in and post lock-in regimes.
\end{abstract}

\section{Introduction}

Vortex induced vibrations (VIV) of circular cylinders is an important area of research in many real life situations including heat exchangers, bridges, tall buildings, riser tubes, towing pipes, power transmission lines etc. VIV, considered and studied as a Fluid Structure Interaction (FSI) problem, demands a crucial attention because of the high amplitude vibrations undergone by the structures which can lead to large scale damages. Flow around circular cylinders stands as the first step in understanding such flows. An important phenomenon which has to be discussed while discussing VIV of cylinders is lockin. When a cylinder is allowed to oscillate freely in a flow, for lower velocities, it oscillates with lower amplitudes whose magnitude varies as per the system parameters. During this stage, the vortex shedding frequency of the cylinder, will be based on the non dimensional quantity, Strouhal number $(S t)$. Strouhal number follows the Strouhal relationship, which is, $S t=\frac{f_{v} D}{U}$, where $f_{v}$ is the vortex shedding frequency at the particular flow velocity, $D$ is the diameter of the cylinder, $U$ is the flow velocity. But as velocity is increased, the response undergoes major qualitative changes. The vortex shedding frequency gets closer to the cylinder's natural frequency and $f_{v}$ gets locked onto $f_{n}$, resulting in a resonance like condition, reflected as high amplitude oscillations for a range of velocities. During lock in, the frequency of vortex shedding is not in accordance with the Strouhal relationship mentioned earlier, but becomes the structure's natural frequency itself. As the velocity is further increased, lockout happens again, with the vortices shedding as per the Strouhal relationship again. There have been numerous

\footnotetext{
^e-mail: aswathymek@gmail.com

$\star \star$ e-mail: sunetra.sarkar@gmail.com
}

amounts of experimental works with focus onto the lockin phenomenon, structural dynamics and the corresponding flow patterns [1], [2], [3], [4]. Experimental studies explain the hydrodynamics part in VIV through explaining the vortex shedding modes and synchronisation regimes in terms of the flow fields [5], [6], [7]. Later, analytical formulations based on 'wake oscillators' gained prominence due to the reason that they are effective in understanding the physics and overall dynamics of the problem through lesser computational efforts [8], [9], [10].

The focus of the present work is to investigate the changes in the dynamics in the presence of noise. A real time system can be subjected to a lot of system uncertainties, which can alter the behaviour of the system as a whole. Studies emphasising the importance of modelling physical systems with the inclusion of noise have been prevalent [11], [12]. Modelling of noise in engineering systems have also gathered attention recently. Triggering mechanism and the role of stochastic noise has been studied for thermoacoustic systems by Waugh et al. [13] and Noiray et al. [14]. Waugh et al. [13] have reported that the intensity of noise has a major role in deciding the practical stability limits. Poirel et al. [15], [16] and Zhao et al. [17] have addressed that aerofoils undergoing flutter with cubic and freeplay nonlinearities are subjected to major qualitative changes when subjected to turbulence in the environment. The number of studies pertaining to discussions on stochastic uncertainties which can be inherently present or externally induced on VIV system are limited. So it is important that we have an understanding on the impact that the fluctuations will have on the system dynamics by studying how big or small changes they can bring onto the response.

In short, we make efforts to capture the role of noise superimposed on an uniform incoming flow in affecting 
the dynamics of an elastically mounted rigid cylinder undergoing transverse oscillations. In the base deterministic model, at each time step, we add a parametric noise modelled through a uniform distribution to the mean flow. Then the system response and their changes are studied with change in the bifurcation parameter. Section 2 explains the formulation of the base deterministic model used for the present study. The bifurcation behaviour of the deterministic system captured using the continuation techniques and direct numerical integration (DNI) are given in section 3. Later, section 4 discusses the stochastic responses obtained using DNI and their qualitative features. In the present work, a low value of noise intensity has been chosen, which is $\sigma=0.1$.

\section{Methodology}

The model consists of a single degree of freedom elastically supported circular cylinder subjected to incompressible constant flow with uniformly distributed noise superimposed on it, as in Figure 1. As described earlier, the physical model is captured using an analytical model where a mechanical spring mass system of the Duffing type models the transverse vibrations of an infinitely long cylinder and a van der Pol oscillator to describe the wake dynamics.

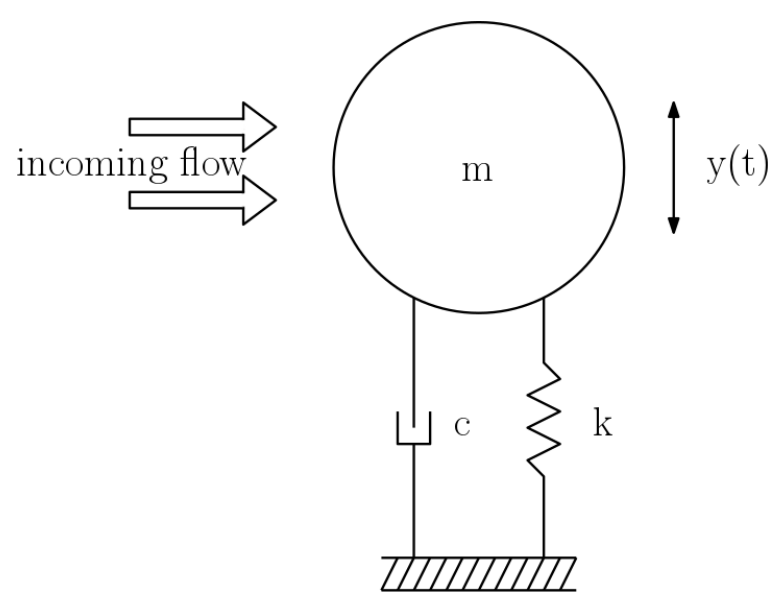

Figure 1: Schematic of cylinder undergoing vortex induced vibrations

The formulation of the model as done by Fachinetti et al. [10] is briefly given as follows. A cubic term has been additionally incorporated in the final structural equation in order to account for the nonlinearities present. The structure oscillator is considered to be a spring mass system oscillating with a single degree of freedom as

$$
m \ddot{Y}+r \dot{Y}+h Y=S
$$

The above equation is given in the dimensional form. Here, the dots denote differentiation with respect to the dimensional time $T, m=m_{s}+m_{f}, r=r_{s}+r_{f}$, where $m_{s}, m_{f}, r_{s}, r_{f}, h, Y, S$ are the structural mass, fluid added mass, structural damping, fluid added damping, spring stiffness, structural displacement, forcing due to the effect of wake, respectively. Here, $m_{f}=\frac{\pi \rho D^{2} C_{M}}{4}$ and $r_{f}=$ $\gamma \Omega_{f} \rho D^{2}$, where $C_{M}$ is the added mass coefficient taken as 1 in case of circular cylinder, $\gamma$ is a stall parameter assumed to be $0.8, \Omega_{f}=\frac{2 \pi S t U}{D}$ is the angular vortex shedding frequency of the cylinder with $S t$ the Strouhal number, $U$ as the incoming flow velocity, $D$ as the diameter of the cylinder. Dividing equation (1) by $m$ gives

$$
\ddot{Y}+\left(\left(2 \zeta \Omega_{s}\right)+\frac{\gamma \mu}{\Omega_{f}}\right)+\Omega_{s}{ }^{2} Y=S / m
$$

with $\mu$ being a non dimensional mass ratio as $\frac{m_{s}+m_{f}}{\rho D^{2}}, \Omega_{s}=$ $\sqrt{\frac{h}{m}}$, being the angular structural natural frequency.

The wake oscillator is modelled by a nonlinear Van der pol equation as

$$
\ddot{q}+\epsilon \Omega_{f}\left(q^{2}-1\right) \dot{q}+\Omega_{f}^{2} q=F
$$

with $F$ being the forcing term on the wake due to the cylinder motion.

Nondimensionalising equations (2) and (3) using $t=$ $T \Omega_{s}$ and $y=Y / D$ and incorporating a cubic term accounting for the geometrical non linearities, as done by Srinil et al. [18], The final forms of non dimensional equations of motion are

$$
\begin{gathered}
\ddot{y}+\left(2 \zeta+\frac{\gamma \Omega}{\mu}\right) \dot{y}+y+\alpha y^{3}=M \Omega^{2} q \\
\ddot{q}+\epsilon \Omega\left(q^{2}-1\right) \dot{q}+\Omega^{2} q=A \ddot{y}
\end{gathered}
$$

In the above equations, overdots mean derivatives with respect to the non dimensional time $t$. Here, $\Omega$ is the ratio of the vortex shedding frequency to the cylinder natural frequency in the medium. It is also defined as $\Omega=S t U_{r}$, where $U_{r}$ is the reduced velocity which is the bifurcation parameter in the present case. The Strouhal number for the present study is assumed to be 0.2 , in the subcritical range of Reynolds numbers [19]. Here, $\mu$ is the mass ratio related to conventional mass ratio as defined by Williamson et al. [7] as $m^{*}=\frac{4 \mu}{p i}-C_{m} ; \epsilon$ and $A$ are empirical coefficients chosen as by [10] as 0.3 and 12 respectively and $\alpha$ is taken as $0.7 . \mathrm{M}$ is a mass number which is a function of the mass ratio defined as $M=\frac{C l_{0}}{16 \pi^{2} S t^{2}}$ [10], with $C l_{0}$ being the associated lift coefficient of a stationary cylinder, taken as 0.3 [19]. The coupling model used for the wake in the present work is an acceleration coupling model [10]. This has been used due to the reason that acceleration coupling model which considers that the structure has a linear inertial effect on the fluid. This coupling model was successful in explaining numerous qualitative aspects of the VIV phenomenon like the lift magnification at lock-in, range of lock-in etc in contrast to the previous coupling models that existed. In the current case, we have used a $m^{*}=248$ and $m^{*} \zeta=0.013$. Further details of the parameters chosen can be referred from [10].

However, a numerical integration of the differential equations alone would not give an exact picture of the type of bifurcations involved, the stability of the trajectories, detection of any unstable branches present etc. In order to 
understand these, we do a continuation study of the set of differential equations. This has been achieved by using the tool XPPAUT [20], which contains codes and algorithms for the continuation and bifurcation software AUTO [21]. Numerical continuation techniques [22] help in finding solutions to a set of parameterised nonlinear equations, from an initial guess for starting the problems. Initially, iterative schemes such as the Newton method are used for solving the system of equations and convergence towards the end solution. The stability of the solutions is determined by calculating eigenvalues and eigenvectors of the fixed points or the Floquet multipliers in case of periodic orbits. Subsequently, this solution is continued to get a curve of solutions of the system for different values of the parameter. Continuation can also be done by starting from a periodic solution. This initial starting orbit is determined by integration and the period of this orbit is used as an approximate period to do continuation by XPPAUT.

\section{Analysis of the deterministic system}

Before studying the stochastic dynamics, it is important to have a clear understanding regarding the dynamics of the deterministic VIV system. We first discuss the bifurcation behaviours and dynamics of the deterministic VIV system obtained using continuation. Later, (DNI) is done to obtain the time histories and frequency spectrums of the structural responses.

Figure 2 shows the bifurcation plot of the VIV system captured using the continuation method. Closed circles show the amplitudes of the stable limit cycles and the open circles show the amplitudes of the unstable limit cycle. Arrows represent the direction of movement of the responses at points where bifurcation of the orbits happen. It is seen that the system is characterised by regions of bistability (two stable solutions for a single parameter) and the steady state solutions of the given system are dependent on the initial conditions given. For example, consider a region consisting of an unstable branch, as in the region between $U_{r}=4.29927$ to $U_{r}=4.77048$. Any initial condition above the unstable branch would take the system to the high amplitude branch, whereas any initial condition below the unstable branch would take it to the low amplitude branch. The unstable solutions become the steady state solution only when our initial condition is also exactly that value. The region between $U_{r}=4.29927$ to $U_{r}=4.77048$ and $U_{r}=5.31756$ to $U_{r}=6.1896$ are regions of hysterisis. The orbit chosen by the system on these regions depends on whether we move in the forward or reverse direction in the bifurcation diagram. It has been understood that the type of bifurcation undergone by the system while transiting from the low amplitude to high amplitude limit cycles is a saddle node bifurcation. These have been confirmed from a Floquet analysis near the bifurcation points and are not shown here for the sake of brevity.

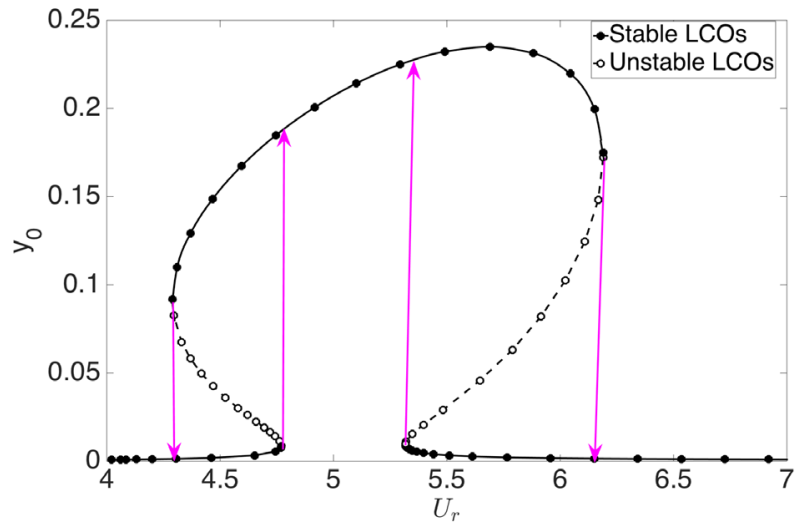

Figure 2: Bifurcation diagram of deterministic system

In the basic formulation of the deterministic model itself, the response $y$ and $q$ are considered to be structural response and wake response respectively with a phase difference, oscillating with a common angular frequency [10]. This common frequency comes out to be the vortex shedding frequency outside lock-in and the structural natural frequency during lock-in. The differential equations have been numerically integrated using a fourth order Runge Kutta method with the bifurcation parameter $U_{r}$ being incremented in steps of 0.1 . As the parameter is increased from $U_{r}=4$, the low amplitude response jumps to a higher branch of response at $U_{r}=4$.8. From $U_{r}=4.8$ to $U_{r}=5.3$, system exhibits high amplitude oscillations. Figure 3 shows the bifurcation diagram obtained by plotting the maximum non dimensional amplitude versus reduced velocity. Figure 4 (a-L) and (a-R) shows time histories and FFTs respectively for $U_{r}=4.7$, figure 4 (b-L) and (b-R) for $U_{r}=4.8$, figure 4 (c-L) and (c-R) for $U_{r}=5.4$, where $\mathrm{L}$ and $\mathrm{R}$ represents left and right sides respectively. The $\mathrm{X}$-axis of the power spectrum plots shows $f$, which is the ratio of oscillation frequency of the response $\left(f_{o}\right)$ to the structural natural frequency $\left(f_{n}\right)$.

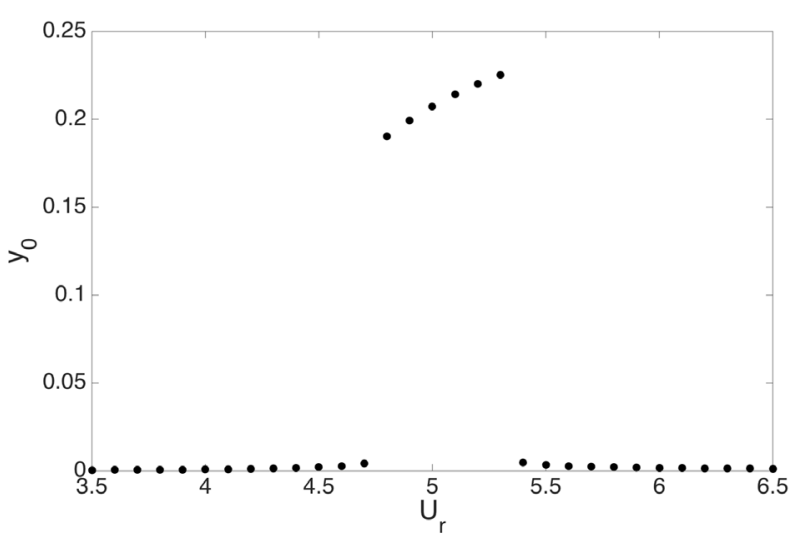

Figure 3: Bifurcation diagram of deterministic system using direct numerical integration 
(a)

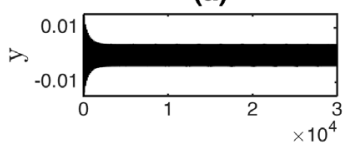

(b)

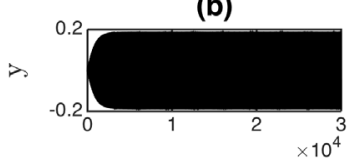

(c)

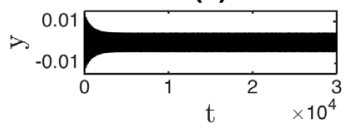

(a)

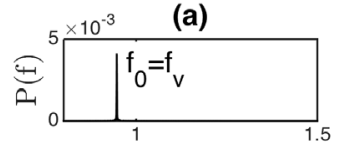

(b)
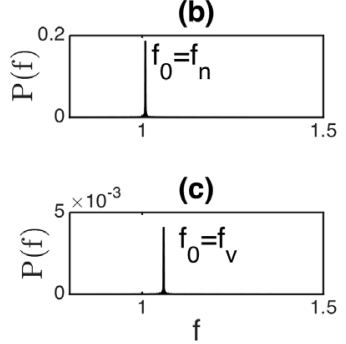

Figure 4: Time histories and FFTs corresponding to the deterministic case for $U_{r}=4.7(\mathrm{a}-\mathrm{L})$ and (a-R), $U_{r}=$ 4.8(b-L) and (b-R), $U_{r}=5.4(\mathrm{c}-\mathrm{L})$ and (c-R)

From Figure 3, it is evident that lock-in occurs from $U_{r}=4.8$ to $U_{r}=5.3$ in the deterministic case. As is seen from the FFTs of Figure 4, all these responses are characterised by single frequencies. At $U_{r}=4.7$, we have small amplitude oscillations of the order of $10^{-3}$, characterised by a single frequency, which is the vortex shedding frequency at this particular velocity. At $U_{r}=4.8$, the oscillations suddenly jump to a high amplitude of order of $10^{-1}(2$ orders higher), again characterised by a single frequency. But this time, the vortex shedding frequency has locked onto the cylinder's frequency and in turn from $U_{r}=4.8$ to $U_{r}=5.3$, the cylinder oscillates with its own natural frequency of oscillation in the fluid. Again at $U_{r}=5.4$, the system has moved out of lock-in, oscillating with very low amplitudes, with the vortex shedding frequency at that particular velocity. As we further increase velocities, it is seen that the oscillation amplitudes get lower though the frequency of oscillation would be the vortex shedding frequencies at the particular velocities.

\section{Noise induced dynamics of the VIV system}

In this section, we investigate the effect of noise on the system dynamics and compare the results with the deterministic cases. The stochastic modelling of velocity is done as follows: The reduced velocity is considered to be a fluctuating parameter, since it is one of the best choices to account for the incoming flow uncertainties. That is, the total reduced velocity at every time considered becomes, $U_{\text {rtotal }}=U_{r}+\sigma u^{\prime}(t)$ where $U_{\text {rtotal }}=$ total reduced velocity, $\sigma$ is the intensity of noise and $u^{\prime}(t)$ is the fluctuating parameter changing at every time step. The input noise fluctuations have been mathematically modelled through a uniform distribution. A distribution like the normal distribution can lead to very large net values and even negative velocity values, which is an unrealistic situation. On the contrary, uniform distribution bounds or limits the maximum value of the net velocity. The values of the fluctuations are determined over a time scale which is very less

compared to the system time scale, through a linear interpolation. That means, the input noise is a rapidly changing one compared to the system responses. Fluid particles will have such high frequency random fluctuations superimposed on their mean velocity generally while operating in the transition regimes to turbulence. These fluctuations can be present not only in the incoming stream, but also in the associated wake of the bluff body, which is encountering vortex shedding. Slight local disturbances around the body, along with the combined effects of the abovementioned effects, might change the dynamics of the FSI problem subjected to transient fluctuations [23].

The output VIV response is analysed for a noise intensity $(\sigma)$ of 0.1 . Figure 5 and Figure 6 show the time histories and FFTs at different values of $U_{r}$. Figure 5 (a) corresponds to the response prior to lock-in, at $U_{r}=4.6$. Comparing these and the deterministic responses prior to lock in discussed previously, it is noticeable that the response is no longer periodic characterised by a single frequency. A Fourier analysis of the structural response shows the existence of two frequencies in the spectrum. One of these frequencies correspond to the vortex shedding frequency itself at the particular velocity. The other frequency seen was found to be corresponding to the structural natural frequency in the oscillating system. These can be seen in Figure 6 (a). That is, the system no longer oscillates with a single frequency, but oscillates as a combination of the inherent system frequencies. The lock-in region is still influenced by the structural dynamics alone, as in the deterministic case, as seen in Figure 6 (b), which is for $U_{r}$ $=5$. Outside lock-in, the system again tries to follow the Strouhal law, following the vortex shedding frequency, but the presence of noise induces a part of structural component in this also, which is seen through the presence of the extra frequency as in Figure 6 (c), for $U_{r}=5.4$. The effects of noise are prominent in the non lock-in states.

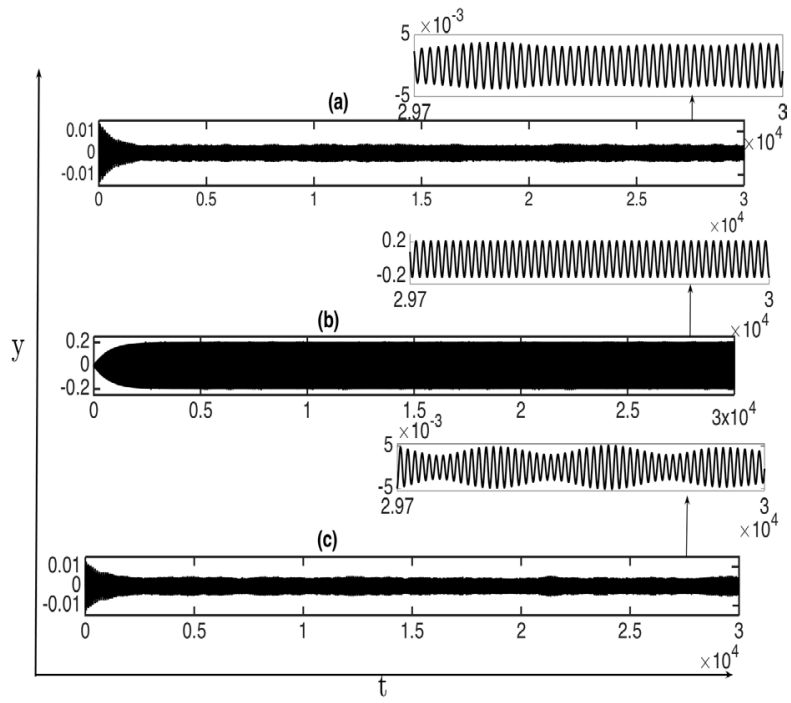

Figure 5: Responses in the presence of noise at $U_{r}$ (a) $=4.6$ [pre lock-in] (b) $=5$ [lock-in] (c) $=5.4$ [just after lock-in] 


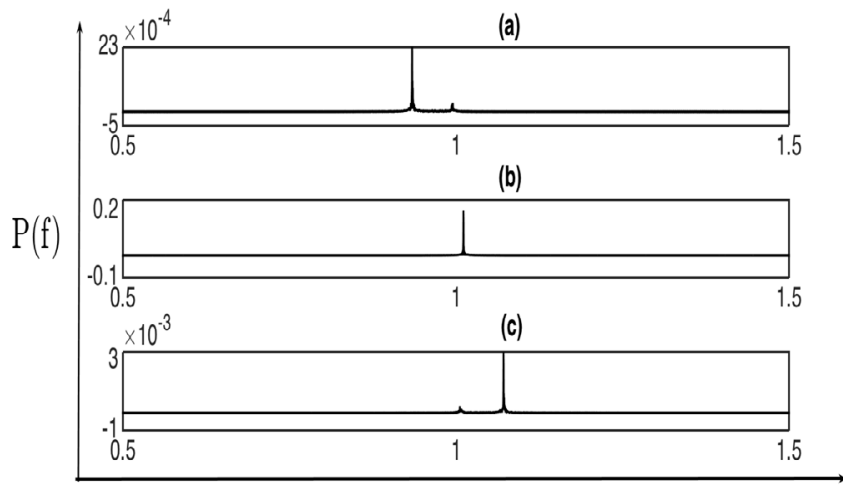

f

Figure 6: Frequency spectrums of the responses in the presence of noise at $U_{r}(\mathrm{a})=4.6$ [pre-lock in] (b) $=5$ [lock in] (c) $=5.4$ [just after lock-in]

The existence of two frequencies under the effect of free stream turbulence has been discussed through experiments by So et al. [24], where a circular cylinder was allowed to undergo cross flow vibrations. They report that the two frequencies correspond to vortex shedding frequency and structural frequency and observe that the two frequencies coalesce at the lock-in point. However, free stream turbulence has been reported to change the Strouhal number post lock-in (unlike in the current work), due to which the domination of the flow or the structural component also changes. In any case, their experiments are consistent with our observations that external noise play a major role in altering the frequency characteristics of the system. Noise seems to act a mechanism through which energy is supplied to the cylinder motion and hence the cylinder's tendency to be in locked-in state remains high. However, the present study has been based on a noise intensity which is very low. The manifestation of frequencies with a higher level of noise intensity might be different from the current scenario. Investigation on these features, along with the identification of the transitional states by a probabilistic approach and through time series analysis are being carried out by the authors presently.

\section{Conclusion}

In the present study, we investigated the effect of stochastic uncertainties in altering the response characteristics of a circular cylinder undergoing Vortex Induced Vibrations. We have observed that uncertainties in the incoming flow can alter the response behaviour considerably both in quantitative and qualitative ways. The responses become fluctuating signals owing to the presence of noise. Noise is seen to invoke additional frequencies in the response oscillations compared to the deterministic cases. The non lock-in states which were characterised by the vortex shedding frequency only in the deterministic cases become subjected to the presence of structural frequency also in the presence of noise. Thus, noise plays a role in driving a part of the system dynamics always controlled by the structure as well. An investigation on whether similar behaviour occurs under a higher noise intensity and studies based on stochastic bifurcations are being pursued by the authors.

\section{References}

[1] P.W. Bearman, Annual review of fluid mechanics 16, 195 (1984)

[2] C.C. Feng, Ph.D. thesis, University of British Columbia (2011)

[3] A. Khalak, C.H.K. Williamson, Journal of Fluids and Structures 11, 973 (1997)

[4] A. Khalak, C.H.K. Williamson, Journal of fluids and Structures 13, 813 (1999)

[5] R. Govardhan, C.H.K. Williamson, Journal of Fluid Mechanics 420, 85 (2000)

[6] A. Khalak, C.H.K. Williamson, Journal of Wind Engineering and Industrial Aerodynamics 69, 341 (1997)

[7] C.H.K. Williamson, Journal of Wind Engineering and Industrial Aerodynamics 69, 3 (1997)

[8] R. Bishop, A. Hassan, The lift and drag forces on a circular cylinder oscillating in a flowing fluid, in Proceedings of the Royal Society of London A: Mathematical, Physical and Engineering Sciences (The Royal Society, 1964), Vol. 277, pp. 51-75

[9] R.T. Hartlen, I.G. Currie, Journal of the Engineering Mechanics Division 96, 577 (1970)

[10] M.L. Facchinetti, E. De Langre, F. Biolley, Journal of Fluids and structures 19, 123 (2004)

[11] L. Arnold, Random dynamical systems (Springer Science \& Business Media, 2013)

[12] W. Horsthemke, in Non-Equilibrium Dynamics in Chemical Systems (Springer, 1984), pp. 150-160

[13] I.C. Waugh, M.P. Juniper, International journal of spray and combustion dynamics 3, 225 (2011)

[14] N. Noiray, A. Denisov (2016)

[15] D. Poirel, S.J. Price, Nonlinear dynamics 48, 423 (2007)

[16] D.C. Poirel, S.J. Price, AIAA journal 39, 1960 (2001)

[17] D. Zhao, Q. Zhang, Y. Tan, Nonlinear Dynamics 58, 643 (2009)

[18] N. Srinil, H. Zanganeh, Ocean Engineering 53, 83 (2012)

[19] R.D. Blevins, Flow-induced vibration (New York, NY (USA); Van Nostrand Reinhold Co., Inc., 1990)

[20] B. Ermentrout, Simulating, analyzing, and animating dynamical systems: a guide to XPPAUT for researchers and students (SIAM, 2002)

[21] E.J. Doedel, A.R. Champneys, T.F. Fairgrieve, Y.A. Kuznetsov, B. Sandstede, X. Wang et al., AUTO97, Concordia University, Canada (1997)

[22] E.L. Allgower, K. Georg, Numerical continuation methods: an introduction, Vol. 13 (Springer Science \& Business Media, 2012) 
[23] G. Buresti, Lecture Notes, International Advanced School on Wind-Excited and Aeroelastic Vibrations, Genoa, Italy (2000)
[24] R.M. So, X. Wang, W.C. Xie, J. Zhu, Journal of Fluids and Structures 24, 481 (2008) 